\title{
Evidence for Rescattering in Intense, Femtosecond Laser Interactions with a Negative Ion
}

\author{
J. Pedregosa-Gutierrez, P. A. Orr, and J. B. Greenwood \\ International Research Centre for Experimental Physics (IRCEP) and School of Maths and Physics, Queen's University Belfast, \\ BT7 1NN, United Kingdom
}

A. Murphy and J.T. Costello

National Centre for Plasma Science and Technology (NCPST) and School of Physical Sciences, Dublin City University, Dublin, Ireland

\author{
K. Zrost, T. Ergler, R. Moshammer, and J. Ullrich \\ Max-Planck-Institut für Kernphysik, D-69029 Heidelberg, Germany
}

(Received 7 June 2004; published 22 November 2004)

\begin{abstract}
It is now well established that energetic electron emission, nonsequential ionization, and high harmonic generation, produced during the interaction of intense, femtosecond laser pulses with atoms (and atomic positive ions), can be explained by invoking rescattering of the active electron in the laser field, the so-called rescattering mechanism. In contrast for negative ions, the role of rescattering has not been established experimentally. By irradiating $\mathrm{F}^{-}$ions with ultrashort laser pulses, $\mathrm{F}^{+}$ion yields as a function of intensity for both linearly and circularly polarized light have been measured. We find that, at intensities well below saturation for $\mathrm{F}^{+}$production by sequential ionization, there is a small but significant enhancement in the yield for the case of linearly polarized light, providing the first clear experimental evidence for the existence of the rescattering mechanism in negative ions.
\end{abstract}

PACS numbers: $32.80 . \mathrm{Gc}, 32.80 . \mathrm{Rm}, 34.80 . \mathrm{Dp}$

The liberation of an electron from an atom immersed in a strong electric field was observed experimentally soon after the discovery that lasers could made to generate moderately intense pulses by $q$-switching them. It was quickly realized that ionization was proceeding by a nonlinear multiphoton absorption process which was easily described within the framework of perturbation theory. However, in the last 20 years the development of ultrashort (femtosecond) pulse lasers has opened up intensity regimes where perturbation theory is no longer valid. In this regime the photon density is so high that ionization proceeds via tunneling of an electron through the barrier created from the superposition of the atomic potential with the macroscopic electric field potential of the laser pulse. The transition from the multiphoton or perturbative to the tunneling or nonperturbative regimes is usually presented in terms of the value of the Keldysh parameter $\gamma[1]$ which is the ratio of the frequency of the radiation to the tunneling rate of the bound electron $[\gamma=$ $\left(I_{P} / 2 U_{P}\right)^{1 / 2}$, where $I_{P}$ is the ionization potential of the electron and $U_{P}$ is the classical ponderomotive energy of a free electron in the electromagnetic field]. Access to the tunneling regime where $\gamma<1$ has resulted in the discovery of many new phenomena. These include enhancements in multiple ionization yields, energetic electron emission, and the generation of high harmonics to very high order.

The universal physical model which has been successfully used to explain these apparently disparate effects is the rescattering or recollision model [2-4]. In this model, the initially ionized electron is considered to be not completely free, rather it propagates in the oscillating laser field. A classical consideration of the electron's trajectory shows that, depending on the phase of the field at the instant the electron is liberated or "born," the electron can either escape with a maximum energy of $2 U_{P}$ or return to the core with an energy of up to3.17U $U_{P}$. An electron which recollides with the ionic core can either recombine yielding a photon of maximum energy $I_{P}+3.17 U_{P}$, cause further ionization by electron impact, or be scattered with an energy of up to $10 U_{P}$, leaving the core unchanged.

The existence of a nonsequential or direct double ionization mechanism has been observed many times as a "knee" in the doubly charged ion yield as a function of laser intensity since it was first reported in 1983 [5]. However, it was not until experiments capable of measuring the ion recoil momentum became possible that recollision could clearly be identified as the main mechanism producing the observed enhancement [6,7]. A particularly sensitive test for the existence of rescattering is a comparison between ion yields obtained using linearly and circularly polarized light. In a circularly polarized field, the electron tends to orbit the core rather than recollide, causing a dramatic suppression of ion yields in double ionization [8].

Despite the impressive progress made to date in the understanding of how atoms behave in intense fields, it has not been shown experimentally if any of these phenomena occur in negative ions. The search for strong field effects in "soft" negative ions is based on much more than simple curiosity. There are expected to be a number 
of significant differences in the behavior of negative ions in strong fields. The short range potential and lower binding energy means the electron's initial wave packet is much larger, thus altering the rescattering amplitude. The electron is also not subject to a Coulomb field as it propagates in the laser field. This "Coulomb focusing" effect has recently been shown to enhance the probability of recollision in atoms [9].

For the analysis of single electron detachment, a Keldysh approach [1] has been reasonably successful in describing the dynamics of the electron emission. A general analytical expression for the differential cross section developed by Gribakin and Kuchiev [10] has been compared with a recent experiment which measured the electron energy and angular distributions in detachment from $F^{-}$[11]. In this study, the existence of electrons with energies greater than the classical cutoff of $2 U_{P}$ could be explained without the need to invoke rescattering which produces a plateau in the electron spectra up to $10 U_{P}$. As the theory treats the initially bound and final continuum states separately (i.e., they are eigenstates of different Hamiltonians), there is no coupling between them and concomitantly no electron-core interaction included.

More recent work, involving a modification of a Keldysh type theory to take into account rescattering [12] and a fully quantum analysis [13], do predict a plateau in the electron spectrum, albeit at energies for which experimental statistics in Ref. [11] were too low. However, comparison of the experimental results with the calculations in Ref. [13] highlighted structure in the electron spectra which could be due to resonant elastic scattering of the electron, which is a common feature in electron-atom collisions [14].

Rescattering effects have also been seen in theoretical work on $\mathrm{H}^{-}$. By making the assumption that the outer electron interacts with only the laser field and the inner electron with the core, Ostrovsky [15] was able to consider the three steps in the rescattering process which lead to excitation of the neutral $\mathrm{H}$ core, and to show that at the intensity studied the direct double detachment rate was considerably higher than the sequential rate. Using an alternative quasienergy, quasistationary approach for solving the Schrödinger equation for the complete system, Frolov et al. [16] showed that a plateau in the electron spectrum exists for $\mathrm{H}^{-}$as well as for $\mathrm{F}^{-}$. While this growing body of theoretical work supports rescattering in negative ions, clear experimental evidence has, to date, remained out of reach.

In the investigation reported here, we have sought out convincing evidence of recollision through measurements of the ion yield arising from double detachment in an atomic negative ion. Fluorine, with an electron affinity of $3.40 \mathrm{eV}$ and an ionization potential of $17.42 \mathrm{eV}$, has been chosen for this study. Its negative ion possesses a closed shell configuration, has a relatively high binding energy, and is amenable to current theoretical methods. Through the differences in the ion yield obtained between linearly and circularly polarized light and their comparison with theoretical yields for sequential ionization, we have been able to definitively demonstrate an enhancement in double detachment for the case of linearly polarized pulses.

The main experimental difficulty in studying recollision in negative ions stems from the fact that neutralization will occur before the intensity of the pulse becomes sufficiently high for excitation or ionization of the core. At the wavelength used for our experiments $(800 \mathrm{~nm})$, the laser intensity required to give a maximum classical recollision energy equal to the first excited state threshold $(12.70 \mathrm{eV})$ is about $6.7 \times 10^{13} \mathrm{~W} \mathrm{~cm}^{-2}$. However, even at lower intensities, when a quantum description of the process is undertaken, excitation or ionization can still proceed if the phases of the core electron and the returning wave packet are favorable. Given that the electron can also initially absorb extra photons through excess photon detachment, double detachment can be produced from recollision at intensities lower than the classical threshold. Theoretical evidence for this can be seen in $\mathrm{H}^{-}$for which recollision excitation of the neutral $\mathrm{H}$ is significant despite the classical recollision energy being a factor of 3 too low [15]. In another study, the time dependent Schrödinger equation was solved directly for $\mathrm{Li}^{-}$. The intensity at which these calculations were made corresponds to a maximum classical return energy which is at least an order of magnitude lower than that needed for excitation. Yet, the authors were able to infer from their results an enhancement in double ionization by recollision, from the observation of an increased rate with a single cycle pulse compared with a double half cycle pulse [17].

By using a pulse length ( $30 \mathrm{fs}$ ) which is about a factor 4 shorter than that used in previous negative ion experiments, we have been able to provide a rapid transition to high intensities. Using the theoretical cross sections [10] for single detachment, we have calculated the expected population of negative ions as a function of time during the laser pulse. The results indicate that $10 \%$ of the ions survive long enough to experience an intensity of $2.5 \times$ $10^{13} \mathrm{~W} \mathrm{~cm}^{-2}$ and $1 \%$ experience $3.5 \times 10^{13} \mathrm{~W} \mathrm{~cm}^{-2}$. Previous experimental results indicate that the theory underestimates the saturation intensity by a factor of 1.76 for $\mathrm{F}^{-}$at $1064 \mathrm{~nm}[10,18]$. If a similar factor is applied here, we expect that a few percent of our ions experienced intensities of $5 \times 10^{13} \mathrm{~W} \mathrm{~cm}^{-2}$. It should be noted that interaction at these intensities corresponds to $\gamma \approx 1$, but as only three photons are required for the removal of the first electron, any double detachment process would occur in the multiphoton regime. The previous experimental study of $\mathrm{F}^{-}$[11] was conducted in the tunneling regime, while the theoretical predictions 
of rescattering enhancement in double detachment were made in the multiphoton regime $[15,17]$.

In our experimental setup [19], the negative ions were produced in a cesium sputter ion source using a $\mathrm{CaF}_{2}$

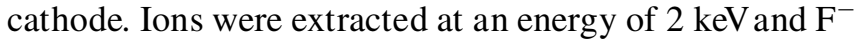
was mass selected by a bending magnet. The beam was collimated and focused into the interaction region in which a pressure of less than $10^{-9}$ mbar was maintained. At the interaction point, a $30 \mathrm{nA}$ beam with a spatial profile of $300 \mu \mathrm{m}$ full width at half maximum was intersected at $90^{\circ}$ by the laser pulses. The $\mathrm{F}^{+}$ions produced were separated from the main beam by deflection plates and detected by an off-axis channel electron multiplier. To minimize the number of $\mathrm{F}^{+}$ions produced from collisions with background gas and scattering from surfaces, the interaction region was voltage labeled to enable rejection of ions produced in other parts of the beam line. The noise contribution from these sources was around 3 counts in $10^{6}$ laser shots.

The laser pulses of wavelength $800 \mathrm{~nm}$ and duration 25 fs were generated by a Ti:sapphire Kerr-lens mode locked oscillator and amplified to an energy of $250 \mu \mathrm{J}$ at a $3 \mathrm{kHz}$ repetition rate. Passage of the beam through a vacuum window was calculated to lengthen the pulse to 30 fs before an on-axis spherical mirror of focal length $100 \mathrm{~mm}$ focussed the light into the ion beam with a focal waist of $15 \mu \mathrm{m}$. For each laser shot there were around 0.1 ions in the laser focus. The radiation was converted from linear to circular polarization by insertion of a quarter wave plate into the output beam and the intensity was controlled by the rotation of a half wave plate placed in front of a linear polarizer.

Figure 1(a) shows the yield of $\mathrm{F}^{+}$ions from linearly and circularly polarized light, normalized to the number of laser shots and the intensity of the ion beam. The intensity scale for the circularly polarized light results has been multiplied by a factor of 0.65 to facilitate a comparison between the two data sets. A theoretical explanation of the origin of this factor is explained later. The error bars shown represent the statistical error in the results at a $68 \%$ confidence interval. As changes in overlap of the two beams could have occurred during the course of a scan due to slight fluctuations in the position and shape of the ion beam, the reproducibility of the measurements over the full range of intensities was checked over the course of the data acquisition. At high intensities, saturation in the sequential removal of the two electrons was demonstrated as the ion yield increases in proportion to $I^{1.5}$ due to the expansion of the focal volume [20].

In Figs. 1(b) and 1(c) the two data sets from Fig. 1(a) are plotted along with theoretical curves for sequential ionization. These were calculated using AmmosovDelone-Krainov (ADK) rates [21] for single ionization of neutral fluorine to obtain an integrated ion yield over the spatial and the temporal profile of the laser focus. The density of the target ions was taken to be constant, which is a good assumption given that the size of the laser focus was much less than that of the ion beam.

To compare results for linear and circularly polarized pulses, the different cycle averaged rates for the two must be considered. If the ion yields as a function of the maximum electric field strengths for each polarization condition are calculated using ADK, the circular yields are higher as the electric field is always "on" during a cycle. If the electric field strength for the circular polarization is multiplied by a factor of 1.14 , the two theoretical curves display excellent agreement in the saturation region. For the experimental data, measurements taken at the same intensity have a maximum electric field strength which is $(2)^{1 / 2}$ lower for the circularly polarized pulses. Therefore, to compare the two data sets as a function of intensity, the intensity scale for the circularly polarized experimental data has been multiplied by $1.14^{2} / 2=0.65$ [22]. The absolute intensity scale for each experimental data set in Figs. 1(a) -1 (c) has been additionally shifted to provide the best fit to the ADK curves. This adjustment lies within the uncertainty in our experimentally measured intensity.

As can be seen in Figs. 1(a)-1(c), there is very good agreement between the two polarization conditions for
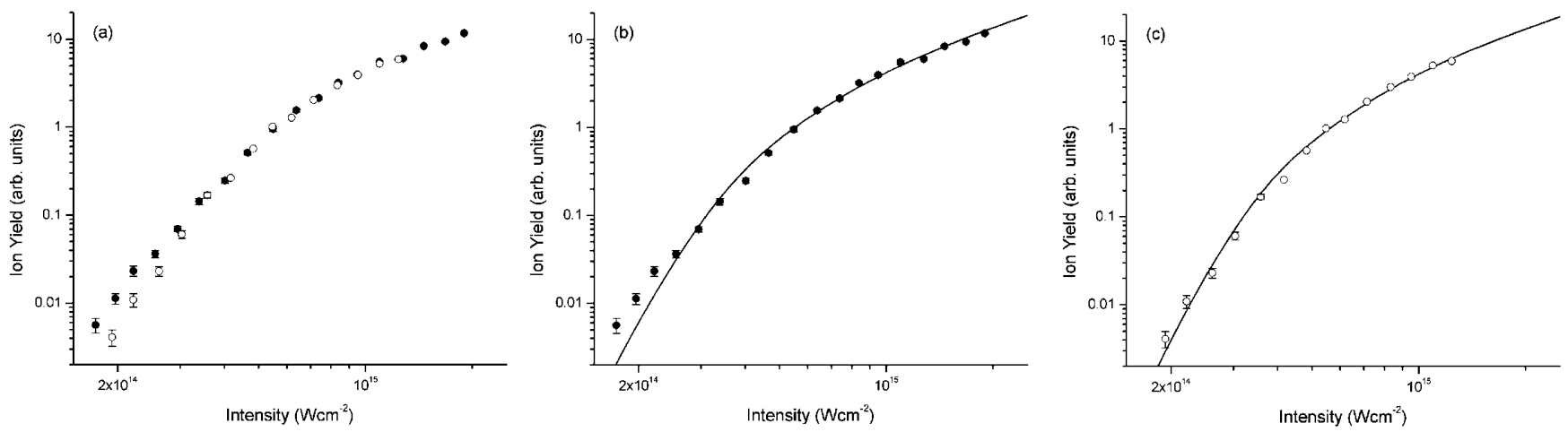

FIG. 1. The yield of $\mathrm{F}^{+}$ions from $\mathrm{F}^{-}$as a function of peak laser intensity: (a) experimental data for linearly (solid circles) and circularly (open circles) polarized light; (b), (c) results for linear and circular polarization, respectively, compared with predictions of ADK theory [21] under the same conditions. 
most of the experimental data and with the theoretical curves. In Fig. 1(c) there is good agreement between the experimental results for circular polarization and the theoretical yields. However, Figs. 1(a) and 1(b) show the results obtained with linear polarization deviate by about a factor of 2 from the circular results and ADK predictions at low intensities. This observation tantalizingly suggests that there is a nonsequential ionization mechanism which enhances the double detachment by linearly polarized pulses. Although we have estimated that only a small fraction of negative ions survive to experience intensities high enough for double detachment by rescattering, this amount is sufficient to explain the enhancement in our signal. While the sequential contribution to the signal is only significant near the peak intensity of the laser focus, the recollision mechanism contributes at much lower intensities and, therefore, the nonsequential yield is derived from a larger interaction volume.

It could be argued that the difference in the sequential rate for linear and circular polarization might also reproduce the experimental results. While there is little difference between the ion yield predicted by ADK theory for the two polarization conditions, this does not account for the possible presence of resonances. For instance, there may be a higher single ionization rate in neutral fluorine for linear polarization if resonances are forbidden in the circularly polarized case. This could occur due to the $\Delta m$ selection rules which increase by one the orbital angular momentum state for the absorption of each circularly polarized photon. The first three excited configurations of fluorine have energies at 12.7, 13.0, and $14.4 \mathrm{eV}$, while between $14.4 \mathrm{eV}$ and the ionization potential of $17.4 \mathrm{eV}$, there are a large number of states which could potentially produce resonances. Given that 12 photons are required to ionize neutral fluorine, increasing to 16 at $10^{14} \mathrm{~W} \mathrm{~cm}^{-2}$ due to channel closing effects, it is unclear if one or two resonances will significantly alter the overall ionization rate. In any case, this type of multiphoton description may not be valid given all our results have been taken in a regime where $\gamma<0.9$ for single ionization of $F$ neutral.

In conclusion, the importance of rescattering in strong field interactions with negative ions has been an open question for some time now due to considerations which suggest that, compared with atoms, this mechanism would be suppressed. These include the short survival time of negative ions in intense pulses and the absence of Coulomb focusing. However, our experimental results provide convincing evidence for a direct double detachment process which is consistent with the rescattering mechanism. This lends support to the recent theoretical work which predicts that rescattering will occur if an intact negative ion can be exposed to a sufficiently strong field [12,13,15-17].

This work was carried out with the support of the Engineering and Physical Science Research Council (UK), the Higher Education Authority (Ireland), Deutsche Forschungsgemeinschaft DFG, and Queen's University Belfast. We would like to thank $\mathrm{H}$. van der Hart, G. F. Gribakin, V. N. Ostrovsky, I. D. Williams, and J. McKenna for their valuable contributions to the discussion.

[1] L. V. Keldysh, Sov. Phys. JETP 20, 1307 (1965).

[2] M. Y. Kuchiev, JETP Lett. 45, 404 (1987).

[3] P. B. Corkum, Phys. Rev. Lett. 71, 1994 (1993).

[4] K. J.Shafer, B. Yang, L. F. DiMauro, and K. C. Kulander, Phys. Rev. Lett. 70, 1599 (1993); K. C. Kulander et al., in Super-Intense Laser-Atom Physics, edited by B. Piraux, A. L'Huillier, and K. Rzazewski (Plenum, New York, 1993), p. 95.

[5] A. LHuillier, L. A. Lompre, G. Mainfray, and C. Manus, Phys. Rev. A 27, 2503 (1983).

[6] T. Weber et al., Phys. Rev. Lett. 84, 443 (2000).

[7] R. Moshammer et al., Phys. Rev. Lett. 84, 447 (2000).

[8] G. D. Gillen, M. A. Walker, and L. D. van Woerkom, Phys. Rev. A 64, 043413 (2001).

[9] V. R. Bhardwaj et al., Phys. Rev. Lett. 86, 3522 (2001).

[10] G. F. Gribakin and M. Y. Kuchiev, J. Phys. B 30, L657 (1997).

[11] I. Y. Kiyan and H. Helm, Phys. Rev. Lett. 90, 183001 (2003).

[12] D. B. Milosevic, A. Gazibegovic, and W. Becker, Phys. Rev. A 68, 050702R (2003).

[13] M.V. Frolov, N. L. Manakov, E. A.Pronin, and A. F. Starace, J. Phys. B 36, L419 (2003).

[14] S. J. Buckman and C.W. Clark, Rev. Mod. Phys. 66, 539 (1994).

[15] V. N. Ostrovsky, J. Phys. B 36, 2647 (2003).

[16] M.V. Frolov, N. L. Manakov, E. A. Pronin, and A. F. Starace, Phys. Rev. Lett. 91, 053003 (2003).

[17] G. L. Kamta and A. F. Starace, J. Mod. Opt. 50, 597 (2003); G. L. Kamta and A. F. Starace, Phys. Rev. A 68, 043413 (2003).

[18] C. Blondel, R-J. Champeau, M. Crance, A. Crubellier, C. Delsart, and D. Marinescu, J. Phys. B 22, 1335 (1989).

[19] J. B. Greenwood, G. F. Collins, J. Pedregosa-Gutierrez, J. McKenna, A. Murphy, and J. T. Costello, J. Phys. B 36, L235 (2003).

[20] M. R. Cervenan and N. R. Isenor, Opt. Commun. 13, 175 (1975).

[21] M.V. Ammosov, N. B. Delone, and V. P. Krainov, Sov. Phys. JETP 64, 1191 (1986).

[22] M. Suresh et al., in Central Laser Facility, Rutherford Appleton Laboratory Annual Report 2004 (unpublished). 